\title{
The GazePlay Project: Open and Free Eye-trackers Games and a Community for People with Multiple Disabilities
}

\author{
Didier SCHWAB ${ }^{1}$, Amela FEJZA ${ }^{1}$, Loïc VIAL ${ }^{1}$, and Yann ROBERT ${ }^{2}$ \\ 1 Univ. Grenoble Alpes, CNRS, Inria, Grenoble INP ${ }^{\star \star}$, LIG, 38000 Grenoble, France \\ \{didier.schwab, loic.vial\}@univ-grenoble-alpes.fr, \\ amela.fejza@grenoble-inp.org \\ 2 Anantaplex \\ yann.robert@anantaplex.fr
}

\begin{abstract}
In order to develop and enhance an augmentative and alternative communication (AAC), gaze is often considered as one of the most natural way and one of the easiest to set up in order to support individuals with multiple disabilities to interact with their environment. For children who start naturally from scratch, who have in addition such difficulties, it is a strong challenge even to acquire or to support the required basic knowledge.

Games are often considered as a good way to learn. Games designed for eye-trackers, i.e. electronic devices able to compute the position of the gaze, allow children to discover the power of their eyes and the consequences of the actions triggered by their gazes. Video games can be a good way to improve basic requirements as gaze fixation and gaze pursuit as well as conventions like rewards or dwell interactions often used in AAC tools. In this article, we present the GazePlay project which main contribution is a free and open-source software which gathers several mini-games playable with any eye-trackers including low cost ones.
\end{abstract}

Keywords: Eye-trackers · Games · Serious Games · Children with Multiple Disabilities · Augmentative and Alternative Communication · HumanComputer Interaction · Eye-based Interaction

\section{Introduction}

In order to develop and enhance an augmentative and alternative communication (AAC), gaze is often considered as being one of the most natural way and one of the easiest to set up in order to support individuals with multiple disabilities to interact with their environment. Of course, for children who start naturally from scratch, who have in addition such difficulties, it is a strong challenge even to acquire or to support the required basic knowledge. They have to learn the power of their eyes, the consequences of the actions triggered by their gazes, they have also to improve their extraocular muscles, etc.

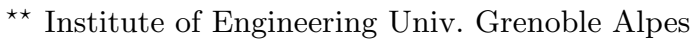


Games are often considered as a good way to learn. In the case of eye-tracking, video games could be a good way to improve basic requirements as gaze fixation and gaze pursuit as well as conventions like rewards or dwell interactions often used in AAC tools.

In this article, we present the GazePlay project which main contributions are

- GazePlay ${ }^{3}$ a free and open-source software which gathers several mini-games playable with any eye-trackers including low cost ones. It permits people to access such games at a price which decreases from $2000-3000 €$ to $100-160 €$ excluding computer price.

- TobiiStreamEngineForJava library ${ }^{4}$, a Java open library which links C++ library from Tobii to Java. With its help, is possible to develop softwares able to interact with Tobii's low-cost eye-trackers (Tobii EyeX, Tobii 4C).

- GazePlay github ${ }^{5}$ a public repository hosted by GitHub which helps us to manage GazePlay development. At the end of March 2018, ten people directly contributed to the code and dozens on ideas (including therapists and parents).

\section{Eye, Gaze and Eye-trackers}

Eye is a sense organ that allows vision. It allows humans to see images in 3 dimensions and with colors. Eyes make specific motions [11][10]:

- Saccade happens when the eyes move from one point of interest to another.

- Pursuit happens when the eyes follow a moving target.

- Fixation happens when the eyes stop scanning the scene, and the vision is kept in one place so it can take detailed information about the object or the scene it is focused on.

- Blinking is a rapid closing then opening of the eyelids. When it occurs spontaneously, it protects the eye by moistening it. When it occurs voluntary, blinking can be used to reestablish a basic communication.

Naked-eye observations go back to the French ophthalmologist Louis Émile Javal (1839-1907), who noticed for the first time that readers eyes do not skim fluently through the text while reading, but make quick movements mixed with short pauses where the eye gets fixated [6]. The very first eye-tracker was created in 1908, again for an experiment about reading. At that time, eye-tracking was really intrusive and uncomfortable for the readers because they had to wear a type of contact lens with a small opening for the pupil. This lens was attached to a pointer which changed its position with the movement of the eye.

Nowadays, eye-trackers are mainly infrared cameras which provide raw coordinates from which it is possible to estimate fixation, saccades, poursuites and/or blinking [8] [4]. Several companies build eye-trackers, for marketing, virtual and

\footnotetext{
${ }^{3}$ https://gazeplay.net

${ }^{4}$ https://github.com/coylz/TobiiStreamEngineForJava

${ }^{5}$ https://github.com/schwabdidier/GazePlay
} 
augmented reality or to evaluate driver vigilance [5]. Eye-based interaction has been used for disabled people since the early 1980's [1] but main work about interaction is [3] where several solutions including dwell interaction which is often used to select in AAC softwares are discussed (see section 3.1).

Until recently, eye-tracker were really expensive devices and were not affordable by the majority of families of disabled people. For instance, in France, the cost of a basic eye-tracker for disabled people is over $1200 €$ in the beginning of 2018 (it was the double one year before).

In 2013, the Eye-Tribe company was the first to extremely lower the cost by creating cheap and more affordable eye-trackers with an elegant Java API and compatible with both Windows and OS X. Tobii, the most important firm to sell eye-trackers released their EyeX $(2014, \simeq 100 €)$ followed by the Tobii $4 \mathrm{C}(2016$, $\simeq 160 €$ ) and their $\mathrm{C}++$ library. While the Eye-tribe was sold to Facebook for its Oculus division and is no longer developped, Tobii works a lot on their low-cost eye-trackers and their associated tools (multiple users, gaze trace, etc.).

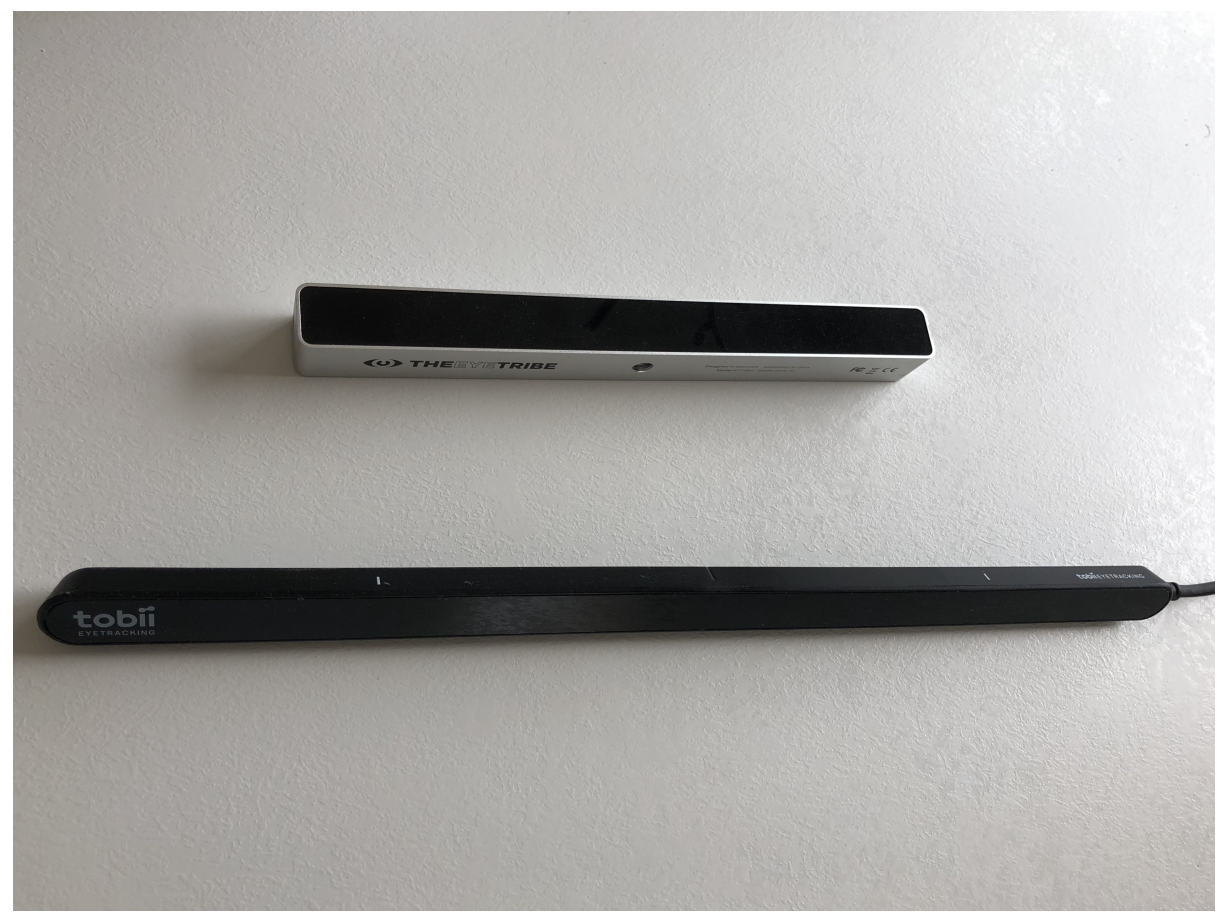

Fig. 1. Two of the eye-trackers which permit to play with GazePlay: the Eye Tribe upper side, the Tobii $4 \mathrm{C}$ bottom side

These three eye-trackers can be used with GazePlay. We integrate directly eye-tribe library while our team has developed a Java Native Interface to build 
a java library, TobiiStreamEngineForJava which permits to use easily the Tobii EyeX and the Tobii $4 \mathrm{C}$ with Java.

\section{GazePlay}

We present in the following GazePlay 1.3, the version of GazePlay we released at the end of March, 2018. GazePlay is a free and open-source software which gathers 22 mini-games playable with an eye-tracker. It is compatible with all eye-trackers which are able to control the mouse cursor, with Tobii EyeX and Tobii 4C on Windows and the Eye Tribe Tracker on Windows or MacOs X. To help motivating the players, it is very easy to modify default images of the game. One player would prefer to play with Mickey Mouse while another would like to play with Princess Sofia, with his family or therapist. Another way to help motivating the players is to give them a reward (a big friendly smiley and applauses). Interface and games are fully translated in French, English and German.

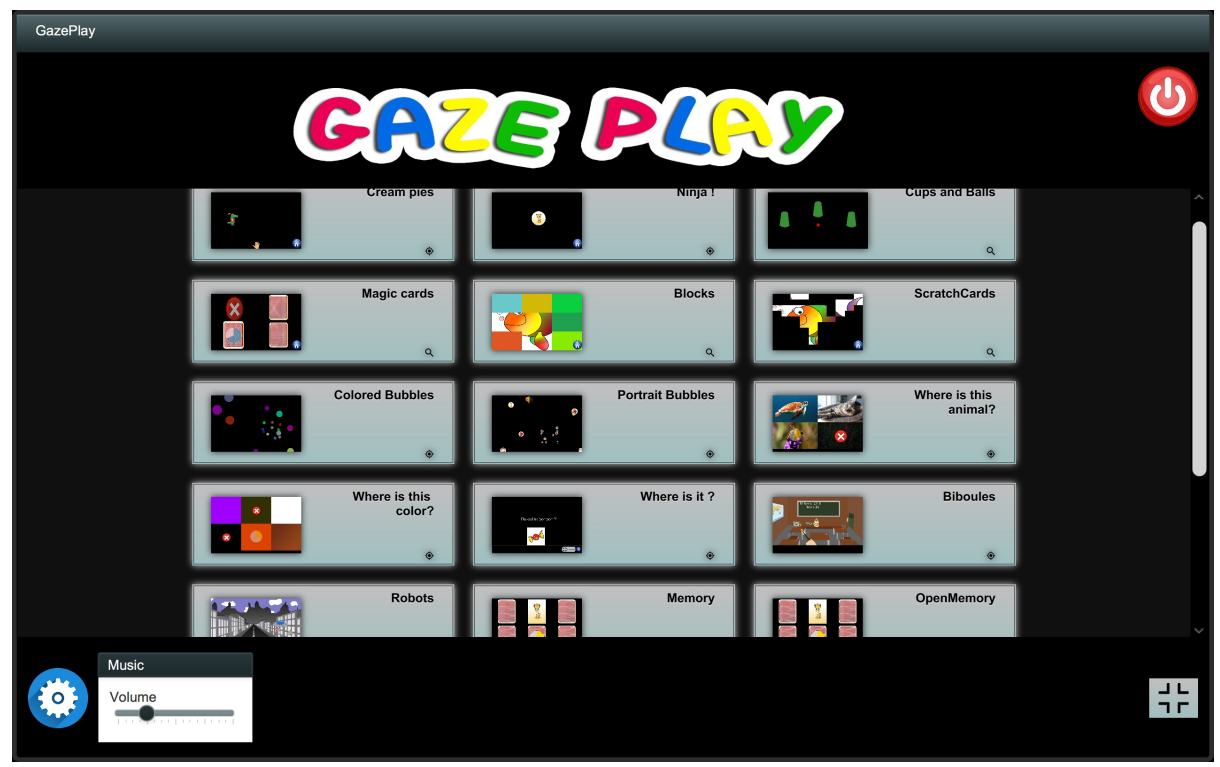

Fig. 2. Screenshot of the main menu of GazePlay development version on the 25th of March, 2018. They are currently 22 games (17 fully playable). 


\subsection{Games in GazePlay}

GazePlay 1.3, gathers 17 playable games ${ }^{6}$. For each one, we aim to develop one or several skills for the children. We can consider that three kinds of skills are developed in GazePlay: action-reaction, selection, memorization skills.

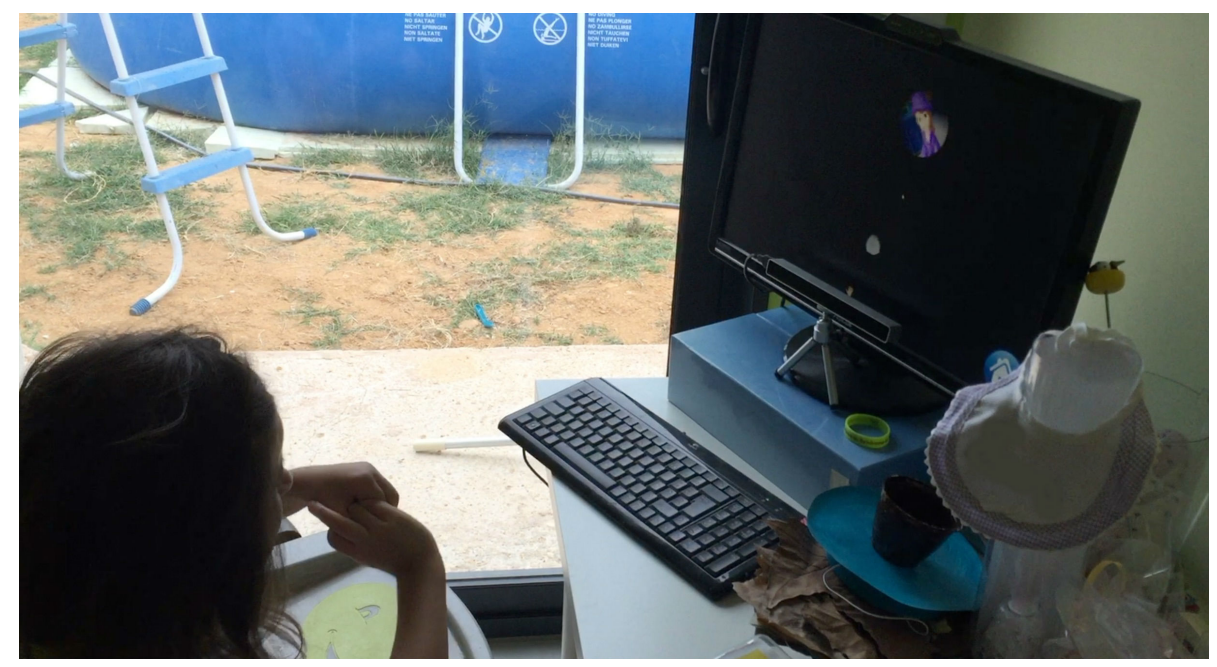

Fig. 3. A child who plays the creampie game with an Eye Tribe tracker.

Action-Reaction Skill The purpose of these games is to teach the children that a gaze can have direct consequences. For instance, in the creampie game, the user throws a custard pie on the target when gaze looks at it (see figure 3) ; in Blocks, a big image is hidden by colored blocks. The player looks at a block to destroy it. When all blocks have disappeared, a reward is shown. In the Ninja game, the target explodes when the player looks at it.

Selection Skill Selection with eye-tracker is not as easy as it is with a mouse. Blinking is both not easy to capture with an eye-tracker and often very difficult to perform for a child with multiple disabilities. In AAC programs, an usual way to select an element (photo, image, pictogram, etc.) is to keep the gaze on this element. It is called, a dwell interaction [3]. A feedback is given as a circular progress bar. When it is completed after a time that can be configured, the element is selected [7]. In Magic Cards several cards are displayed. One of the card hides an image. When the player stares at one card, it is turned. If (s)he finds the image, a reward is given to the player. Other games like Memory and Where is it? aim at training the selection skill.

\footnotetext{
${ }^{6}$ For a complete description of games see https://gazeplayeng.wordpress.com/games/
} 
Memorization Skill The purpose of these games is to work on the short-term memory like in memory which has the same rules as the ones of the classic game. Several pairs of cards from 2 to 6 (according to the child/helper choice), face down. Then the player uses a dwell interaction (see previous section) to turn over 2 cards. If both cards are the same, they disappear, otherwise they turn back over after a few seconds to allow the player to remember them. When all cards have been matched, the reward is given.

In other games, we aim at working on long-term memory. In the game Where is the animal?, a sentence is pronounced and written to indicate to the player which animal to find (horse, dog, crocodile,...) and then several photos of animals are shown (from 4 to 9 according to the child/helper choice). Player selects photos until (s)he finds the correct one. The same game is proposed with colors. An additional one is completely configurable ie. parents/therapists can build their own games with their own photos. The sentence can be completed with pictograms for instance to teach them to the children (see figure 4). This game and the use of pictograms was inspired by the community (see section 4).

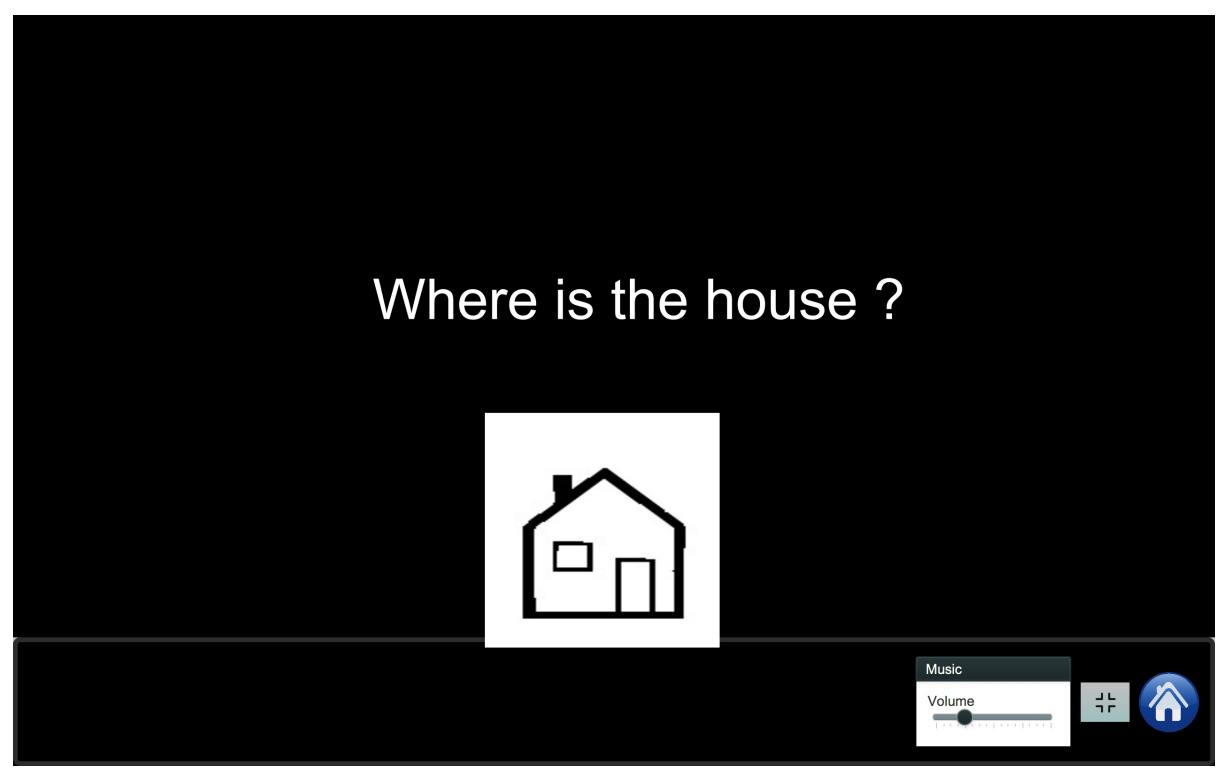

Fig. 4. Screenshot of the game where is it?, the question is asked associated to a pictogram from Makaton vocabulary [2].

\subsection{Analysis}

When a game is stopped by the helper, statistics are displayed and saved automatically. They include charts which present depending on the game, total active 
playtime, actual playtime, durations, reaction length, standard deviation, etc. A heatmap also shows gaze position on the screen. With these statistics, helpers get objective information to evaluate motivation and/or evolution of children.

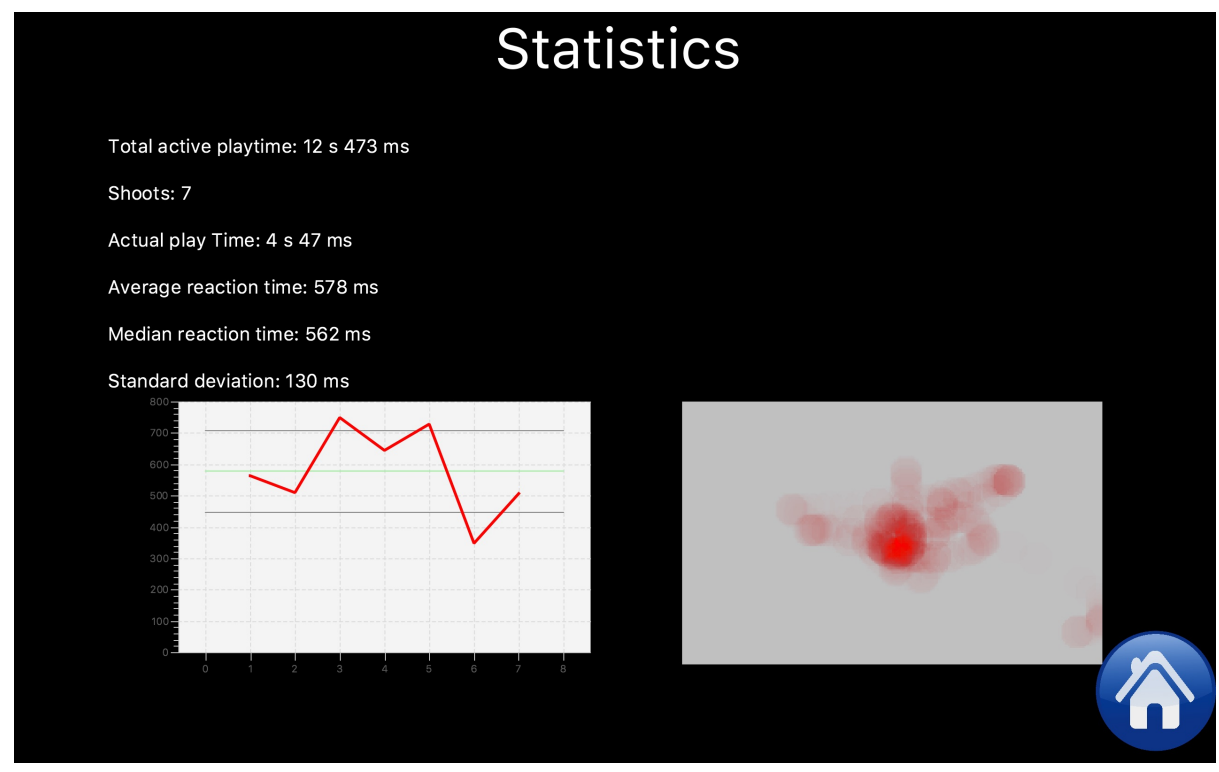

Fig. 5. Screenshot of statistics for an action-reaction focused game.

\section{The Community as the Future of GazePlay}

The project is open source under a GNU General Public License v3.0 ${ }^{7}$. Anyone can modify it as long as (s)he distributes hi(s)her code. It remains possible to sell customized version of GazePlay or services using GazePlay including therapies or tutorials.

The GazePlay project started with the first author who is both an associate professor in informatics and the father of a girl with multiple disabilities. Sharing experience with other parents especially the ones who already own eye-trackers and games for their children and with therapists [9], he worked with postgraduate students (second and third authors) to release first versions of GazePlay.

The code is hosted on GitHub. The repository permits to share code, information for developers, issues (enhancements, bugs, milestones, etc) but also to find interesting projects. Due to his goal, some people (fourth author for instance) from the open source community helped us especially on the software

\footnotetext{
7 https://www.gnu.org/licenses/gpl-3.0.en.html
} 
engineering side and permited to increase our efficiency. Similarly, we get regular feedback from many therapists who used GazePlay with children.

GazePlay was downloaded almost 800 times since its first release. We really hope that such a project can inspire others to build new projects related to people with special needs.

\section{References}

1. Friedman, M.B., Kiliany, G., Dzmura, M.: The eyetracker communication system. Johns Hopkins APL Technical Digest 3(3), 250-252 (1982)

2. Grove, N., Walker, M.: The makaton vocabulary: Using manual signs and graphic symbols to develop interpersonal communication. Augmentative and Alternative Communication 6(1), 15-28 (1990). https://doi.org/10.1080/07434619012331275284, https://doi.org/10.1080/07434619012331275284

3. Jacob, R.J.K.: What you look at is what you get: Eye movement-based interaction techniques. In: Proceedings of the SIGCHI Conference on Human Factors in Computing Systems. pp. 11-18. CHI '90, ACM, New York, NY, USA (1990). https://doi.org/10.1145/97243.97246, http://doi.acm.org/10.1145/97243.97246

4. Jambon, F., Luengo, V.: Analyse oculométrique " on-line " avec zones d'intérêt dynamiques : application aux environnements d'apprentissage sur simulateur. In: Régis Mollard, Franck Poirier, F.V. (ed.) Ergo'IHM 2012 - Conférence sur les nouvelles interactions, créativité et usages. p. publication en cours. Biarritz, France (Oct 2012), https://hal.archives-ouvertes.fr/hal-00873898, session plénière Ergo/IHM/IS

5. Kar, A., Corcoran, P.: A review and analysis of eye-gaze estimation systems, algorithms and performance evaluation methods in consumer platforms. IEEE Access 5, 16495-16519 (2017). https://doi.org/10.1109/ACCESS.2017.2735633

6. Lupu, R.G., Ungureanu, F.: A survey of eye tracking methods and applications (2014)

7. Nguyen, V.B., Jambon, F., Calvary, G.: Gaze-Based Interaction: Evaluation of Progressive Feedback. pp. 153-158. ACM (Oct 2014), https://hal.archivesouvertes.fr/hal-01090436, poster

8. Salvucci, D.D., Goldberg, J.H.: Identifying fixations and saccades in eye-tracking protocols. In: Proceedings of the 2000 Symposium on Eye Tracking Research \& Applications. pp. 71-78. ETRA '00, ACM, New York, NY, USA (2000). https://doi.org/10.1145/355017.355028, http://doi.acm.org/10.1145/355017.355028

9. Schwab, D.: Gazeplay : Creation of a community to help the development of a free and open-source plateform to make eye-tracker video games accessible to everyone. In: 5ème EUROPEAN RETT-SYNDROME CONGRESS. Berlin (November 2017)

10. Tatler, B.W., Wade, N.J., Kwan, H., Findlay, J.M., Velichkovsky, B.M.: Yarbus, eye movements, and vision. iPerception 1(1), 7-27 (2010). https://doi.org/10.1068/i0382, http://www.ncbi.nlm.nih.gov/pmc/articles/PMC3563050/

11. Yarbus, A.L.: Eye Movements and Vision. Plenum. New York. (1967) 